\title{
Aula invertida: experiencia en el Grado de Enfermería
}

\author{
Flipped classroom: nursing degree experience
}

\section{$M^{a}$ Pilar Sabater-Mateu ${ }^{1}$, Jorge Juan Curto-García ${ }^{2}$, Àngela Rourera-Roca, $M^{a}$ Carmen Olivé-Ferrer ${ }^{3}$, Silvia Costa-Abós ${ }^{4}$, Sara Castillo-Ibáñez, Amparo del Pino-Gutiérrez}

\author{
Departament d'Infermeria de Salut Pública, Salut Mental i Maternoinfantil. \\ Facultat de Medicina i Ciències de la Salut \\ Universitat de Barcelona \\ Campus Bellvitge - Pavelló de Govern, Feixa Llarga, s/n, 08907, L’Hospitalet del Llobregat \\ psabater@ub.edu jicurto@ub.edu angelarourera@ub.edu \\ olivecarmina@ub.edu scosta@ub.edu castillo.ibanez@gmail.com \\ adelpino@ub.edu
}

\begin{abstract}
Resumen
En el aula invertida, los estudiantes trabajan autónomamente tareas previamente preparadas con este fin por el profesorado. Objetivo: evaluar el aula invertida como innovación docente y contrastar los resultados con grupo con docencia tradicional. Contexto: primer curso del Grado de Enfermería en la asignatura troncal Ciencias Psicosociales Aplicadas a la Salud (grupo $\mathrm{C}, \mathrm{n}=81$ ). Metodología: cualitativa y cuantitativa con la inclusión de instrumentos estandarizados ad hoc. Destaca la evaluación por pares y contrastes verbales sobre contenidos tutelados por el profesorado. Se realizó evaluación del grupo control y el grupo experimental y se contrastaron los resultados de las cualificaciones obtenidas. Resultados. Puntuación sobre 10 con medias y desviación estándar: Intraclase, superior en el aula invertida $(7,96 \pm 0,67)$ frente a $(5,89 \pm 1,26)$ en examen (p-valor $<0,001)$. Interclase, grupo experimental $(5,89 \pm 1,26)$ y grupo control $(6,02+1,16)$, (p-valor 0,508$)$. Sin hallazgo de diferencias estadísticamente significativas. Conclusión: Sin preparación para el examen y gran esfuerzo adicional, destacó la satisfacción por parte del profesorado y de los estudiantes, el aula invertida se mostró claramente favorable para la adquisición de conocimientos en la asignatura de Enfermería probada.
\end{abstract}

Palabras claves: Aula Invertida, Competencia comunicativa, Competencia trabajo en equipo, Evaluación formativa, Grado de Enfermería, Ciencias Psicosociales Aplicadas a la Salud.

\begin{abstract}
At flipped classroom, students work autonomously with tasks previously prepared for this purpose by the professorship. Objective: To evaluate the flipped classroom as innovation teaching and contrast group results with traditional teaching. Context: first course of Nursing Degree at Psychosocial Sciences Applied to Health, core subject (Group $\mathrm{C}, \mathrm{n}=81$ ). Methodology: qualitative and quantitative, including ad hoc standardized instruments and emphasizing the evaluation performed by peers and verbal verification on the content. The whole process was supervised by lecturers. Evaluation: We conducted the evaluation of the control group and the experimental group, and they compare the results of the rating results. Results: Score out of 10 with means and standard deviation: Intraclass, higher flipped classroom $(7,96+0,67)$ vs $(5,89+1,26)$ in test ( $p$-value $<0,001)$. Interclass, experimental group $(5,89+1,26)$ and control group $(6,02+1,16)$ (p-value 0,508$)$. Statistically significant differences were not

\footnotetext{
1 Responsable del Grup d'Innovació Docent Consolidat (GIDC): Grup d'Infermeria Orientat a Tècniques Educatives Innovadores. Universitat de Barcelona (GIOTEI-UB). España.

${ }^{2}$ Miembro del GIOTEI-UB.

${ }^{3}$ Miembro del GIOTEI-UB.

${ }^{4}$ Miembro del GIOTEI-UB.

${ }_{5}^{5}$ Corresponding author. Miembro del GIOTEI-UB.
} 
found. Conclusion: Without test preparation and great additional effort, highlighted the satisfaction for lecturers and students, flipped classroom was clearly favorable for the acquisition of knowledge in the subject tested.

Key words: Collaborative learning, Flipped classroom, Formative assessment, Nursing Degree, Active learning methodologies, Psychosocial Sciences Applied to Health.

\section{Introducción}

En el aula invertida, los estudiantes trabajan de forma autónoma tareas previamente preparadas con este fin por el profesorado (Jordan et al., 2014). Esta metodología permite mejorar el aprendizaje de los alumnos mediante el intercambio de roles profesor-alumno (Jiménez, 2013). En nuestro caso, el alumno inicia el estudio de la materia fuera del aula y lo profundiza en una sesión presencial en la clase con la discusión con el resto de compañeros y el apoyo del profesor.

Estudios publicados internacionalmente desde disciplinas diversas dentro de las ciencias sociales y el campo de la salud, entre otras, Harrington et al. (2015), Jensen et al. (2015), Jordan et al. (2014), Missildine et al. (2013), Rodríguez et al. (2015), Schwartz (2014) y Simpson y Richards (2015), así como otra experiencia piloto que realizamos con anterioridad (curso académico 2013-14) en nuestra misma asignatura, indican que el aula invertida presenta ventajas como un aprendizaje más profundo, la adquisición de competencias transversales y la motivación del alumnado en el aula. No obstante, diversos autores refieren también que presenta aspectos que pueden dificultar su implementación (Jordan et al., 2015 y Missildine et al., 2013). Un ejemplo puede ser el caso de la planificación y preparación previa de material (Schwartz, 2014), necesaria por parte del profesor. Así mismo, ha de considerarse la ardua evaluación continuada por parte del docente y el trabajo autónomo del estudiante fuera y dentro del aula. Todo ello puede ocasionar la falta de aceptación generalizada de los estudiantes por este método (Missildine et al., 2013, Rodríguez et al., 2015, Simpson y Richards, 2015 y Strayer, 2007).

En el presente trabajo analizamos la metodología de aula invertida a partir de un proyecto de innovación docente, reconocido por el Programa de Millora i Innovació Docent (PMID) de la Universitat de Barcelona (UB), (Referencia: 2014PID-UB / 052). Se trata de una experiencia realizada en la asignatura troncal (9ECTS) "Ciencias Psicosociales Aplicadas a la Salud" de primer curso del Grado de Enfermería de la UB durante el curso académico 2014-15.

La necesidad de llevar a cabo la actuación docente innovadora, se consideró por parte del profesorado a partir de la detección de la distracción frecuente de los estudiantes con las nuevas tecnologías (por ej. teléfonos móviles) y la consecuente reducción de motivación y de presencialidad en clase. Así, se diseñó y planificó una nueva modalidad de clases más proactivas en sustitución de las clases teóricas tradicionales o magistrales, que se tradujo en la modalidad de aula invertida cuya experiencia se presenta.

Así mismo, se contempló que la asignatura de Ciencias Psicosociales Aplicadas a la Salud tiene una base de conocimientos teóricos que necesariamente el estudiante ha de consolidar para poder aplicarlos durante el periodo de socialización asistencial. También se tuvo en cuenta, que en el contexto de primer curso, cuando se imparte esta asignatura, los estudiantes no han iniciado sus prácticas clínicas. En suma, los contenidos de dicha asignatura en el aula pueden resultar especialmente abstractos (por ejemplo se explica el tipo de relación de ayuda a establecer con el paciente y su familia, cuando aún no ha habido ocasión de acudir a prácticas a un centro asistencial) y verse fortalecidos desde un enfoque docente que potencie la proactividad.

En este sentido, se ha apuntado que la modalidad de docencia tradicional complementada con trabajos prácticos y seminarios, no evita que parte del conocimiento resulte abstracto y su estudio resulte excesivamente memorístico hasta avanzar a posteriores períodos curriculares (Gibbs y Simpson, 2009). 
Por ello, mediante el aula invertida se buscó incidir en el autoaprendizaje a partir de bibliografía y trabajo guiado como método para dar respuesta a las preguntas planteadas en la asignatura.

En consecuencia, se buscó la sincronización del aprendizaje de conocimientos dentro y fuera de clase e incentivar la presencialidad en el aula, mediante la aportación de contenidos escritos y una dinámica participativa que incluyera la discusión y el debate. En dicho marco docente, se han favorecido las habilidades comunicativas escritas y orales, se ha buscado inducir al estudiante al pensamiento crítico y complejo e incidir en el modelado de actitudes a través del discurso (mediante presentaciones grupales y opiniones colectivas) a través del tutelaje de la profesora. Simultáneamente, se ha potenciado el trabajo en equipo, a la vez que retroalimentar la enseñanza-aprendizaje en un escenario que permite la actuación docente in time on time, además del recurso de la modalidad virtual.

Incorporado el material docente en el campus virtual de la asignatura, por esta vía se han gestionado, particularmente, resultados de la evaluación continuada a cargo de la profesora. Dicha evaluación ha consistido en la publicación de registros semanales (fruto de la corrección de trabajos escritos individuales y grupales) con indicación de déficits detectados y acciones consecutivas a emprender por el/los estudiantes. Todo este conjunto, ha repercutido en la mejora de la competencia de autorregulación del estudiante (conocer el producto de su trabajo, organizarse y responsabilizarse en consecuencia). Así, el estudiante, más allá de la memorización pudo favorecer la comprensión y consolidación de contenidos de aspectos psicosociales del cuidar.

\section{Objetivos}

Se realizó este estudio con metodología de innovación docente con el objetivo de favorecer la motivación y el aprendizaje proactivo de los estudiantes. Los objetivos específicos fueron hacia los estudiantes y hacia el profesorado buscando promover el aprendizaje proactivo de conocimientos dentro y fuera de la clase, incentivar la presencialidad, la dinámica y el debate en clase, favorecer habilidades comunicativas escritas y verbales, inducir al estudiante al pensamiento crítico y complejo, incidir en el modelado de actitudes a través del discurso, presentaciones grupales, opiniones colectivas y tutelaje de la profesora, potenciar el trabajo en equipo, retroalimentar la enseñanza-aprendizaje en tiempo no presencial (mediación virtual) y en modalidad presencial o en tiempo real, evaluar de forma continuada (evaluaciones semanales publicadas en campus virtual, con indicación de déficits individuales y grupales detectados y acciones consecutivas correspondientes, y por último favorecer la competencia de autorregulación del estudiante (organizarse y responsabilizarse de su trabajo). Así mismo se buscó dinamizar la clase y promover el interés de los estudiantes por aprender, favorecer en el estudiante la interrelación de contenidos de la asignatura y su comprensión duradera en el tiempo (evitar sólo memorizar), incitar al contraste de conocimientos a través del trabajo grupal, además de disponer de un escenario presencial para la práctica y evaluación del trabajo en equipo.

\section{Metodología}

Para poder realizar la nueva metodología docente, ésta se explicó en el aula y se puso en conocimiento de los alumnos. Se estableció en el grupo C, constituido por 81 estudiantes, del turno de tarde y segundo semestre del curso académico 2014-15. Se apuntó que el programa de seguimiento era el propio de la asignatura Ciencias Psicosociales Aplicadas a la Salud, impartido en el primer curso del grado de Enfermería en la Universidad de Barcelona. Tras las aclaraciones pertinentes se obtuvo el consenso total de los estudiantes para su implementación.

Los contenidos explicativos del nuevo proyecto fueron: 1) Presentación de tres vídeos: uno sobre cambios educativos en el siglo XXI, y dos de breves sobre concepto de aula invertida. 2) Plan del nuevo método y de su dinámica: guía didáctica, conjunto de preguntas temáticas, fichas de respuesta (individual) y plantillas de evaluación (individual y para su empleo en evaluación por pares); 3) 
evaluación a cargo de la profesora: puntuación, frecuencia (semanal), ubicación (en campus virtual) recomendaciones derivadas para el alumno (reparación de correcciones). 4) Distribución del tiempo en clase y sincronización con otras actividades de la asignatura (seminarios y trabajos).

Acerca de otras actividades de la asignatura, la modalidad de aula invertida planteada, suponía mantener los seminarios y la presentación de trabajos, comunes al resto de grupos de la asignatura (A, B, D, Cprimer semestre) y su misma tónica en temporalidad y dinámica.

Respecto a la evolución general de la asignatura, se planteó la posibilidad opcional, de supresión de la prueba objetiva de conocimientos (incorporada como obligatoria en las clases tradicionales), si se superaban las tareas encomendadas en la modalidad aula invertida. Dicha prueba suponía un valor del $30 \%$ de la puntuación total de la asignatura.

La estructura de distribución de tiempo durante las dos horas semanales de clase fue la siguiente:

$1^{\text {a }}$ hora de clase: Dinámica del aula invertida. Distribución de tiempo en tres etapas de duración 20'x20'x20', con el desglose siguiente: 20 minutos para evaluación por pares (del trabajo escrito realizado fuera del aula); 20 minutos de debate en pequeño grupo, para hacer un resumen consensuado del conjunto de respuestas aportadas al tema por los miembros del grupo; 20 minutos de exposición oral de resúmenes ante la clase, a través del coordinador del grupo (cargo rotatorio semanal elegido por los estudiantes) y aclaraciones a nivel de grupo grande con intervenciones de la profesora.

$2^{a}$ hora de clase: Presentación de trabajos (por ej. trabajo grupal de casos, entre otros), o realización de seminarios según pautas comunes para el resto de grupos de la asignatura.

En consecuencia, el conjunto de actividades de innovación realizadas por el alumno, le supuso semanalmente: a) contestar por escrito preguntas y resumirlas en una ficha individual estandarizada (fuera del aula); b) evaluar por pares ficha individual anterior, corrigiendo y registrando déficits en la plantilla individual, creada con este fin (en clase); c) participar en la discusión del pequeño grupo. El fin era consensuar un resumen grupal de los contenidos escritos en la ficha individual para exponerlos oralmente en clase, el coordinador del grupo.

En cuanto a la calificación a obtener por el alumno, la respuesta adecuada de la ficha puntuó un máximo de 10 puntos. Del conjunto de 15 fichas y temas trabajados se extrajo la nota media, suponiendo el máximo de esta el $30 \%$ de la nota final de toda la asignatura. A este resultado se añadieron las calificaciones obtenidas en seminarios y otros trabajos de la asignatura.

Así mismo, se permitió modificar los contenidos de la ficha para su adecuación hasta un máximo de tres veces. Repetir parte o la totalidad de la ficha, reducía su puntuación máxima a una nota de 5 puntos. No presentar la ficha, o no presentarla adecuadamente, aportaba 0 puntos. Así el trabajo del estudiante integró indicadores cualitativos y cuantitativos.

Otras puntuaciones fueron las atribuidas mediante la prueba objetiva de conocimientos (examen tipo test), que buscó avalar con notas y sus contrastes, la evaluación intraclase e interclase del aula invertida implementada. Por tanto, dicha evaluación integro indicadores cuantitativos y se agregaron otros cualitativos fruto de la opinión de los estudiantes, acerca de la satisfacción obtenida con el plan de innovación docente desarrollado.

El balance del material y estrategia docente seguida en su conjunto es que posibilitó una evaluación semanal de calidad: por pares y grupal (en clase y por parte de estudiantes) y una segunda ronda también individual y grupal (por parte de la profesora) que genero la evaluación publicada en el campus virtual con indicaciones de incidencias y acciones a desarrollar por el estudiante, contemplando y cumpliendo, en conjunto, criterios de claridad y transparencia. 
Cabe resaltar que la esencia de la modalidad de el aula invertida planteada, supone que el trabajo básico de leer y preparar un primer texto escrito con bibliografía individual y semanal, el estudiante lo realiza de forma autónoma fuera de clase. Con lo cual, el tiempo presencial en el aula, puede ser aprovechado para revisar, corregir, analizar, contrastar, debatir y consensuar el conocimiento adquirido, que finalmente se transcribe en la ficha grupal prevista para tal actividad. Todo este proceso, apunta que el tiempo en clase es rentabilizado para trabajar estrategias cognitivas de nivel superior (por ej. contrastar, discriminar, seleccionar...) en base a la taxonomía de Bloom (Gibbs y Simpson, 2009).

\section{Resultados}

Resultados intraclase. Sobre una puntuación máxima de 10, la nota media en modalidad aula invertida fue de 7,96 $(\mathrm{n}=81)$. De estos estudiantes, 78 hicieron la prueba objetiva escrita obteniendo una nota media de 5,89, sin hallarse diferencias estadísticamente significativas entre ambas evaluaciones ( $p$ $<0.001$, test de Wilcoxon) como se puede comprobar en la Tabla 1.

\begin{tabular}{|l|c|c|c|c|}
\hline & $\mathrm{n}$ & Media \pm DE & Mediana (Min-Max) & \multirow{2}{*}{ p-valor } \\
\hline Nota aula invertida (sobre 10) & 81 & $7,96 \pm 0,67$ & $8,13(5,80-8,90)$ & \multirow{2}{*}{$<0,001$} \\
\hline Nota examen (sobre 10) & 78 & $5,89 \pm 1,26$ & $5,95(1,00-8,50)$ & \\
\hline Diferencia nota el aula invertida -examen & 78 & $2,08 \pm 1,35$ & $2,10(-1,97-7,13)$ & \\
\hline DE - Desviación Estandar
\end{tabular}

DE - Desviación Estandar

${ }^{*}$ Test de Wilcoxon

Tabla 1: Resultados intraclase. Nota el aula invertida, prueba objetiva escrita y diferencia

En cuanto a las notas categorizadas en los 81 estudiantes evaluados a través del método aula invertida sobre una puntuación de 10, el porcentaje de aprobados con nota entre 5-8 fue de 42,0\% (n=34) y de $78,2 \%(\mathrm{n}=61)$ para los 78 alumnos que hicieron el examen. En cuanto a la nota entre 8-10 en el método aula invertida fue de $58 \%(\mathrm{n}=47)$, mientras que en el caso de los que hicieron la prueba objetiva escrita el porcentaje fue de $5,1 \%(n=4)$. Suspendieron el 16,7\% $(n=13)$ estudiantes. En consecuencia, como se puede observar en la Tabla 2, se encontraron diferencias estadísticamente significativas entre las evaluaciones ( $\mathrm{p}<0,001$, prueba de homogeneidad marginal).

\begin{tabular}{|c|c|c|c|}
\hline & & $\mathrm{n}(\%)$ & p-valor* \\
\hline \multirow{3}{*}{ Nota aula invertida } & Aprobado 5-8 & $34(42,0)$ & \multirow{7}{*}{$<0,001$} \\
\hline & Aprobado 8-10 & $47(58,0)$ & \\
\hline & Total & $81(100,0)$ & \\
\hline \multirow{4}{*}{ Nota examen } & Suspenso & $13(16,7)$ & \\
\hline & Aprobado 5-8 & $61(78,2)$ & \\
\hline & Aprobado 8-10 & $4(5,1)$ & \\
\hline & Total & $78(100,0)$ & \\
\hline
\end{tabular}

* Prueba de homogeneidad marginal

Tabla 2: Resultados categorizados. Nota el aula invertida y prueba objetiva escrita

Una vez analizadas ambas evaluaciones con el coeficiente de correlación de Pearson, no se encontró una correlación estadísticamente significativa entre ellas (coeficiente de correlación de Pearson $=0,106, p$ valor $=0,356$ ).

Resultados interclase. Según se ha indicado anteriormente, se dispuso de la nota de 78 estudiantes evaluados a través del método aula invertida, que además hicieron la prueba objetiva escrita, obteniendo una nota (media \pm DE $5,89 \pm 1,26$ ) y de 98 estudiantes del grupo control que obtuvieron una nota (media 
$\pm \mathrm{DE} 6,02 \pm 1,16)$. No encontrándose diferencias estadísticamente significativas entre ambos grupos ( $\mathrm{p}=0,508$, prueba $t$ para muestras independientes), como puede observarse en la Tabla 3 .

\begin{tabular}{|c|c|c|c|c|}
\hline $\begin{array}{l}\text { Nota examen } \\
\text { (sobre 10) }\end{array}$ & $\mathrm{n}$ & Media $\pm \mathrm{DE}$ & Mediana(Min-Max) & p-valor* \\
\hline $\mathrm{C}$ & 78 & $5,89 \pm 1,26$ & $5,95(1,00-8,50)$ & \multirow{2}{*}{0,508} \\
\hline $\mathrm{A}$ & 98 & $6,02+1,16$ & $6,20(3,30-8,50)$ & \\
\hline
\end{tabular}

Tabla 3: Resultados en el Grupo aula invertida (interclase). Nota aula invertida, prueba objetiva escrita y diferencia con grupo control

En cuanto al contraste de notas categorizadas en los dos grupos: experimental (C) y control (A) el porcentaje de aprobados con una nota entre 5 y 8 en los 78 estudiantes del grupo aula invertida, fue del $78,2 \%(n=61)$, mientras que en el grupo control fue del 79,6\% $(n=78)$. Las notas entre 8-10 fueron de $5,1 \%(\mathrm{n}=4)$ para el grupo aula invertida y de $2,0 \%(\mathrm{n}=2)$ para el grupo control. Las notas con suspenso fueron de $16,7 \%(\mathrm{n}=13)$ para el grupo aula invertida y de $18,4 \%(\mathrm{n}=18)$ para el grupo control. No se encontraron diferencias estadísticamente significativas entre los grupos en el porcentaje de estudiantes aprobados ( $\mathrm{p}=0,598$, Test exacto de Fisher) como podemos ver en la Tabla 4.

\begin{tabular}{|c|c|c|c|}
\hline & Grupo C & Grupo A & \\
\hline Nota examen sobre 10 & $\mathrm{n} \mathrm{( \% )}$ & $\mathrm{n}(\%)$ & p-valor* \\
\hline Suspenso & $13(16,7)$ & $18(18,4)$ & \multirow{2}{*}{0,598} \\
\hline Aprobado 5-8 & $61(78,2)$ & $78(79,6)$ & \\
\hline Aprobado 8-10 & $4(5,1)$ & $2(2,0)$ & \\
\hline *Test exacto de Fisher & & \\
\end{tabular}

Tabla 4: Notas de examen según grupo (categorizadas)

\section{Conclusiones}

En general, se puede concluir que los objetivos alcanzados en el desarrollo del proyecto fueron los previstos en el diseño de la innovación docente. Como aspecto adicional, se vio necesario realizar un cierto seguimiento individual y/o grupal de un sector de estudiantes (en torno a un 15\%) a través de tutoría. Fue un grupo de alumnos que por causas laborales, familiares o personales (por ej. problemas de salud), tuvieron un ritmo más lento de adaptación a la nueva dinámica docente, que el resto de la clase. Esta situación repercutió en el contenido y/o formas de entrega del material elaborado por el estudiante fuera del aula. Estos aspectos se fueron subsanando con el seguimiento docente apuntado anteriormente. En este sentido, en concordancia con diversos autores, este trabajo concluye que este método no es igualmente efectivo para todo tipo de estudiantes, ya que exige madurez al discente, un control sobre su aprendizaje y un tipo de compromiso, al cual, en ocasiones no están acostumbrados el alumnado (Jiménez, 2013). En suma, se trata de un método que busca elementos que garanticen la dedicación y el compromiso a este aprendizaje (Simpson y Richards, 2015 y Jordan et al., 2014), aún más ante la posibilidad de eximir al alumno de ciertas pruebas, como puede ser un examen en nuestro caso.

Así, en la experiencia desarrollada destacan fundamentalmente tres aspectos nucleares que avalan el valor atribuible a esta innovación docente: calificaciones intraclase, calificaciones interclase y cantidad de trabajo implícito en la modalidad de clase invertida diseñada. De este conjunto puede extraerse un balance final del proyecto contrastado con argumentos como los siguientes: 
Calificaciones intraclase. Los estudiantes del grupo experimental obtuvieron por el método del aula invertida en torno a dos puntos más de media, o calificaciones más altas que las que obtuvieron a través de la prueba objetiva de conocimientos (examen tipo test). Lo que equivale a interpretar que los conocimientos adquiridos eran equivalentes o superiores a los contenidos de un examen tradicional para la materia. Al respecto, se ha apuntado que las investigaciones cuantitativas de los resultados de estudiantes con el método de aula invertida son muy limitados (Harrington et al., 2015), aunque destaca el caso de una tesis doctoral realizada en EEUU desde esta perspectiva (Jiménez, 2013). Por otro lado, la nota superior del aula invertida es común en otros estudios y es una de las características que se relaciona con este método. Así pues, se ha apuntado que los estudiantes sacan mejores notas que con la modalidad tradicional según estudios realizados entre estudiantes de enfermería (Missildine et al., 2013) y de farmacia (Wong et al., 2014), entre otros ejemplos (Jiménez, 2013).

Calificaciones Interclase. Teniendo en cuenta las condiciones descritas en el apartado de resultados el número de suspensos fue inferior en el grupo experimental $(16,7 \%)$ que en el caso del grupo control. $(18,4 \%)$. El número de calificaciones con puntuación entre 8-10 (sobre 10) fue también superior en grupo experimental $(5,1 \%)$ frente al grupo control $(2,0 \%)$. Finalmente, las diferencias entre aprobados en la franja 5-8 fueron reducidas y suponen 78,2\% en grupo experimental frente a 79,6\% en grupo control. En consecuencia, resulta bastante evidente, que los conocimientos adquiridos mediante el método del aula invertida, resultaron favorables para el conjunto de los estudiantes que siguió la experiencia.

En otros trabajos publicados, también se da el caso de poca diferencia entre los tipos de notas procedentes de la modalidad aula invertida y modelo tradicional, como por ejemplo, en el caso de enfermería (Harrington et al., 2015) y en el caso de estudios de biología (Jensen et al, 2015).

Cantidad de trabajo implícito en la modalidad de aula invertida diseñada. Para un grupo de 80 estudiantes el trabajo desarrollado desde la perspectiva docente es desmedido. Es también muy laboriosa la planificación previa de material estandarizado, aunque es profundamente superado por la evaluación semanal de los 80 estudiantes, individual y en grupo. Este exceso de trabajo, incluida la preparación previa, ha sido reconocido también en otros casos (Schwartz, 2014) aun contando con grupos más reducidos, como por ejemplo, en torno a 50 alumnos estudiantes de enfermería (Missildine et al., 2013). Además, en algún estudio también se ha contemplado la carga externa de trabajo (de otras asignaturas) como factor que puede dificultar la implementación de este método (Jordan et al., 2015).

Por parte de los estudiantes, se recopilo información sobre su satisfacción con el método y aprendizaje obtenido. En su mayoría reconocieron más trabajo que en la modalidad tradicional (clase magistral, junto con seminarios, trabajos y examen). El exceso de trabajo planteado como detractor del método, ha sido reconocido por otros estudiantes y publicaciones (Simpson y Richards, 2015). En otros casos, se prefiere claramente la modalidad tradicional, por ejemplo en matemáticas (Gibbs y Simpson, 2009) o también en estudiantes de enfermería (Missildine et al., 2013), así como en medicina (Rodríguez et al., 2015), aunque en ocasiones, la diferencia de preferencias sobre uno de los dos métodos sea mínima, como sucedió en otro grupo de estudiantes de enfermería (Simpson y Richards, 2015). Desde nuestro contexto, este aspecto podría ilustrarse con la opinión de un estudiante que al respecto escribió: "Desgraciadamente no tenemos esta cultura de trabajo" (Jiménez, 2013).

Por otro lado, también en su mayor parte los estudiantes apuntaron más y mayor consolidación de conocimientos que era uno de los propósitos principales del proyecto. Estudiantes de farmacia ensalzaron también las bondades y preferencias por el método de aula invertida (Wong et al., 2014) al igual que estudiantes universitarios de lengua inglesa (Jiménez, 2013) aunque se reconocen opiniones en los dos sentidos, como sucede en nuestro caso. Nuestro grupo, de forma particular, destacó el interés por el trabajo en equipo, aspecto enfatizado igualmente por estudiantes del grado de medicina (Rodríguez et al., 2015). 
Un aspecto especialmente destacable de la experiencia de aula invertida desarrollada, es que presentó congruencia entre los conocimientos que se esperaba que los estudiantes aprendieran y/o comprendieran y los que finalmente mostraron haber aprendido junto con su interrelación desde la óptica de la perspectiva compleja. En ello pudo influenciar la metodología, en base al trabajo autónomo del estudiante y las posibilidades interactivas reiteradas (discusión en grupo, presentación en clase, etc....), junto a su implicación y responsabilidad. Obviamente, esta apreciación puede verse dificultada cuando el conocimiento complejo se mide mediante pruebas más afines al pensamiento lineal, o causa-efecto, como puede suceder con determinadas pruebas objetivas de conocimiento, entre las que podría contarse por ejemplo, el caso del examen tipo test.

Finalmente, en cuanto a las posibilidades de transferir la experiencia desarrollada de aula invertida, desde la perspectiva metodológica, incluido el material docente estandarizado diseñado ad hoc, parece perfectamente plausible su extrapolación a experiencias docentes de otros grados según se deduce también de otros estudios publicados. De forma particular, grados o asignaturas vinculados con las ciencias de la salud y también relacionados con las ciencias sociales o las humanidades. Para el profesorado, una clave seria tener entre las aspiraciones docentes impulsar en el estudiante la integración de conocimientos y su interrelación, perspectiva notoriamente destacada en el desarrollo competencial. Además, cabe tener en cuenta que el aula invertida se reconoce internacionalmente, como una estrategia eficaz para la educación (Harrington et al., 2015).

\section{Agradecimientos}

Al Programa de Millora i Innovació Docent de la Universitat de Barcelona, por la ayuda económica concedida al proyecto. Proyecto de Innovación Docente, Referencia: 2014PID-UB/052.

\section{Referencias}

Gibbs, G. y Simpson, C. (2009) Condicions per una avaluació continuada que afavoreixi l'aprenentatge. ICE i Editorial Octaedro. Universitat de Barcelona.

Harrington, S.A., Vanden Bosch, M., Schoofs, N., Beel.Bates, C., Anderson, K. (2015) Quantitative Outcomes for Nursing Students in a Flipped Classroom. Nursing Education Perspectives, 36(3), pp. 179-181.

Jensen, J.L., Kummer, T.A., Godoy, P.D.d.M. (2015) Improvements from a Flipped Classroom May Simply Be the Fruits os Active Learning. CBE-Life Sciences Education, 14, pp. 1-12.

Jordan Lluch, C., Pérez Peñalver, M.J., Sanabria Codesal, E. (2015) Educación inversa, una metodología innovadora ¿Coincide la percepción que tienen los alumnos de ella con la nuestra? XIII Jornadas de Redes de Investigación en Docencia Universitaria Nuevas estrategias organizativas y metodológicas en la formación universitaria para responder a la necesidad de adaptación y cambio. Universidad de Alicante. Recurso electrónico recuperado 30/10/2016 (https://rua.ua.es/dspace/bitstream/10045/49558/2/XIII_Jornadas_Redes_148_poster.pdf).

Jordan Lluch, C., Pérez Peñalver, M.J., Sanabria Codesal, E. (2014) Experiencias docentes. Investigación del impacto en un aula de matemáticas al utilizar flip education. Revista Pensamiento matemático, IV(2), pp. 09-022.

Jiménez Muñoz, AJ. (2013). Auge y caída de la clase al revés: ¿Lecciones aprendidas? Recurso electrónico recuperado 30/10/2016

(https://www.academia.edu/7420281/Auge_y_ca\%C3\%ADda_de_la_clase_al_rev\%C3\%A9s_lecciones aprendidas ?auto=download).

Missildine, K., Fountain, R., Summers, L., Gosselin, K. (2013) Flipping the Classroom to Improve Student Performance and Satisfaction. Journal of Nursing Education, 52(19), pp. 597-599. 
Rodríguez Learte, A.I., Fernández Vaquero, A., Vega Avelaira, D. (2015) Desarrollo de metodologías de “flipped classroom" para asignaturas de ciencias básicas: valoración de los alumnos. XII Jornadas Internacionales de Innovación Universitaria. Educar para transformar: Aprendizaje experiencial, pp. 692-698.

Schwartz, T.A. (2014) Flipping the Statistics Classroom in Nursing Education. Journal of Nursing Education, 53(4), pp. 199-206.

Simpson, V., Richards, E. (2015) Flipping the classroom to teach population health: Increasing the relevance. Nurse Education in Practice, 15, pp. 162-167

Strayer, J.F. (2007) The effects of the classroom flip on the learning environment: a comparison of learning activity in a traditional classroom and a Flip Classroom that used an intelligent tutoring system. Dissertation Presented: Degree Doctor of Philosophy in the Graduate School of The Ohio State University (Dr. Douglas T. Owens, Adviser). Recurso electrónico recuperado 30/10/2016

(https://etd.ohiolink.edu/rws_etd/document/get/osu1189523914/inline).

Wong, T.H., Ip, E.J., Lopes, I., Rajagopalan, V. (2014) Pharmacy Students' Performance and Perceptions in a Flipped Teaching. Pilot on Cardiac Arrhythmias. American Journal of Pharmaceutical Education, 78(10), pp. 1-6. 